University of Nebraska - Lincoln

DigitalCommons@University of Nebraska - Lincoln

6-14-2007

\title{
Effects of magnetostatic coupling on stripe domain structures in magnetic multilayers with perpendicular anisotropy
}

\author{
K. Janicka \\ University of Nebraska - Lincoln \\ John D. Burton \\ University of Nebraska-Lincoln, jburton2@unl.edu \\ Evgeny Y. Tsymbal \\ University of Nebraska-Lincoln, tsymbal@unl.edu
}

Follow this and additional works at: https://digitalcommons.unl.edu/cmrafacpub

Part of the Nanoscience and Nanotechnology Commons

Janicka, K.; Burton, John D.; and Tsymbal, Evgeny Y., "Effects of magnetostatic coupling on stripe domain structures in magnetic multilayers with perpendicular anisotropy" (2007). Faculty Publications from Nebraska Center for Materials and Nanoscience. 38.

https://digitalcommons.unl.edu/cmrafacpub/38

This Article is brought to you for free and open access by the Materials and Nanoscience, Nebraska Center for (NCMN) at DigitalCommons@University of Nebraska - Lincoln. It has been accepted for inclusion in Faculty Publications from Nebraska Center for Materials and Nanoscience by an authorized administrator of DigitalCommons@University of Nebraska - Lincoln. 


\title{
Effects of magnetostatic coupling on stripe domain structures in magnetic multilayers with perpendicular anisotropy
}

\author{
K. Janicka, ${ }^{\text {a) }}$ J. D. Burton, and E. Y. Tsymbal \\ Department of Physics and Astronomy, Nebraska Center for Materials and Nanoscience, \\ University of Nebraska, Lincoln, Nebraska 68588-0111
}

(Received 7 March 2007; accepted 26 April 2007; published online 14 June 2007)

\begin{abstract}
We investigate the effects of magnetostatic interlayer coupling on the formation of magnetic domains in exchange-coupled ferromagnetic films exhibiting perpendicular magnetic anisotropy. Using simple models for the stripe domain structures we investigate how the magnetostatic interlayer interaction affects the domain size. For antiferromagnetically exchange-coupled multilayers, we show that there is a domain overlap due to the magnetostatic interaction which exists only above a critical thickness and below a critical separation between the ferromagnetic films where it is scaled inversely proportional to the antiferromagnetic exchange coupling constant. We show that the magnetostatic coupling is responsible for the periodic "tiger tail" domain structure observed experimentally and demonstrate the dependence of the period of this structure on the interlayer exchange coupling. (c) 2007 American Institute of Physics. [DOI: 10.1063/1.2745376]
\end{abstract}

\section{INTRODUCTION}

Thin-film structures composed of ferromagnetic thin films separated by a metallic or an insulating nonmagnetic spacer layer have aroused significant interest. Such systems exhibit giant magnetoresistance (GMR) or tunneling magnetoresistance (TMR) phenomena, making them attractive for applications as magnetic field sensors and magnetic randomaccess memories (for reviews on GMR and TMR see Refs. 1 and 2, respectively). Functioning of these magnetoresistive devices is very sensitive to magnetic interactions between the layers. Therefore, tremendous efforts have been invested in the understanding of the mechanisms controlling these interactions.

One of them is the interlayer exchange coupling (IEC) which has been thoroughly investigated in the past two decades (for reviews of IEC see Refs. 3 and 4). It was found that the magnetizations of two ferromagnetic thin films separated by a nonmagnetic metallic spacer layer are coupled via an exchange interaction mediated by the itinerant electrons of the spacer layer. ${ }^{5}$ In this case the IEC oscillates between ferromagnetic and antiferromagnetic as a function of the nonmagnetic layer thickness, ${ }^{6-8}$ the periods of the oscillations being determined by critical vectors spanning the Fermi surface. ${ }^{9}$ The IEC is explained by the formation of quantumwell states in the spacer layer ${ }^{10}$ and originates from the quantum interference of Bloch waves due to spin-dependent reflections at the ferromagnet/paramagnet interfaces ${ }^{4}$ but has essentially the same origin as the Ruderman-Kittel-KasuyaYosida (RKKY) interaction between localized magnetic moments in a nonmagnetic host metal. ${ }^{4}$

If two ferromagnetic films are separated by an insulating barrier layer the mechanism of IEC is the tunneling spin polarization propagating through the barrier. ${ }^{11,12}$ Unlike a metallic spacer layer, the insulating layer leads to nonoscillatory coupling which decays exponentially as a function of

${ }^{a)}$ Electronic mail: janicka@bigred.unl.edu the barrier thickness reflecting the evanescent nature of the states mediating the exchange. ${ }^{13,14}$ In this case, the strength and the sign of the interlayer coupling are very sensitive to impurity and defect states in the barrier. ${ }^{15,16}$

If two ferromagnets are separated by an antiferromagnetic spacer layer the IEC is controlled by the antiferromagnetic order in the spacer layer. For example, it was found that $[\mathrm{Co} / \mathrm{Pt}] / \mathrm{NiO} /[\mathrm{Co} / \mathrm{Pt}]$ multilayers exhibit oscillatory IEC. ${ }^{17,18}$ The period of oscillation corresponds to the antiferromagnetic ordering period of the $\mathrm{NiO}$, suggesting a correlation of the coupling with the antiferromagnetic order. ${ }^{19,20}$

Another kind of the interlayer coupling may occur due to magnetostatic interactions between the ferromagnetic layers. For example, correlated roughness at the ferromagnet-spacer interfaces results in the Néel coupling, ${ }^{21}$ affecting properties of magnetoresistive devices. ${ }^{22,23}$ Uncorrelated interface roughness may produce a biquadratic coupling which has a tendency to align the magnetizations of the two ferromagnetic layers perpendicular to one another. ${ }^{24}$ It is well known that interface properties, ${ }^{25}$ including roughness, ${ }^{26}$ largely control transport characteristics of magnetoresistive devices and therefore their accurate description is critical for the understanding of device performance.

Magnetostatic stray fields play an important role in nonuniformly magnetized films. The magnetostatic interlayer coupling is responsible for a progressive reduction of the remnant magnetization of a hard ferromagnetic layer by repeated switching of a neighboring soft layer. ${ }^{27}$ Even under moderate fields, mirrored domains may be formed in the hard and soft layers due to stray fields ${ }^{28}$ The stray fields in one magnetic layer lower the nucleation field in the other layer due to domain walls. ${ }^{29}$ Understanding the role of magnetostatic interactions in magnetic layered structures is critical for the development of advanced magnetoresistive devices and recording media.

The magnetostatic coupling affects the formation of domains in exchange-coupled ferromagnetic multilayers exhib- 
iting perpendicular magnetic anisotropy. The interest in magnetic thin-film layered structures with perpendicular anisotropy has been stimulated by the fact that they are expected to improve density, stability, and reliability of spin valves and magnetic tunnel junctions. ${ }^{30}$ Magnetic thin films with perpendicular anisotropy produce stripe domain structures, ${ }^{31}$ so that magnetostatic stray fields have a tendency to align magnetic domain moments parallel to one another, i.e., contribute to ferromagnetic interlayer coupling. For antiferromagnetically exchange-coupled multilayers, this magnetostatic coupling competes with the interlayer exchange interaction resulting in unusual domain structures. For example, it was found that CoPt films exchange-coupled antiferromagnetically through $\mathrm{Ru}$ (Ref. 32) layers exhibit a relative shift between the domains of the two magnetic layers. A similar behavior was observed for CoPt films separated by an antiferromagnetic $\mathrm{NiO}$ spacer layer. ${ }^{33}$ Furthermore, it was found that the orientation of the parallel-aligned magnetization regions reverses periodically along the domain wall, ${ }^{32,33}$ producing a "tiger tail" structure and indicating a more complex mechanism of the domain formation in these multilayers.

Stimulated by these experimental findings, we investigate the effects of the magnetostatic coupling on the formation of domains in exchange-coupled ferromagnetic films exhibiting perpendicular magnetic anisotropy. Using simple models for the stripe domain structures we demonstrate that there is an effect of the magnetostatic interlayer coupling on the domain size in magnetic multilayers with perpendicular anisotropy. For antiferromagnetically exchange-coupled multilayers, we demonstrate that the domain overlap due to the magnetostatic interaction exists only above a critical thickness of ferromagnetic films where it is scaled inversely proportional to the antiferromagnetic exchange coupling constant. We show that the magnetostatic coupling is also responsible for the tiger tail domain structure observed experimentally. ${ }^{32,33}$

\section{DOMAIN SIZE OF A FERROMAGNETICALLY COUPLED MULTILAYER}

It is known that magnetostatic interactions influence the domain size in magnetic thin films with perpendicular anisotropy where the magnetization is aligned perpendicular to the plane producing stripe domain structures. For films with thickness much greater than the domain width, the stripe domain size follows Kittel's law which predicts the proportionality between the square of the domain width $L^{2}$ and the thickness of a film $t .{ }^{31}$ The origin of this behavior is a competition between the magnetostatic energy and the domain wall energy. For such films the magnetostatic energy per unit volume associated with the domains is proportional to the domain size $L$ and inversely proportional to film thickness $t .^{31}$ The domain wall energy per unit film volume is inversely proportional to $L$, reflecting the density of domain walls. According to Kittel the former can be written as $E_{m} / 2$ $\simeq 0.85 M^{2} L / t$, where $M$ is the saturation magnetization of the film. The latter can be expressed as $E_{\mathrm{dw}}=\sigma / L$, where $\sigma$ is the domain wall energy per unit area. Minimizing the total energy $E=E_{m}+E_{\mathrm{dw}}$ leads to an equilibrium domain width

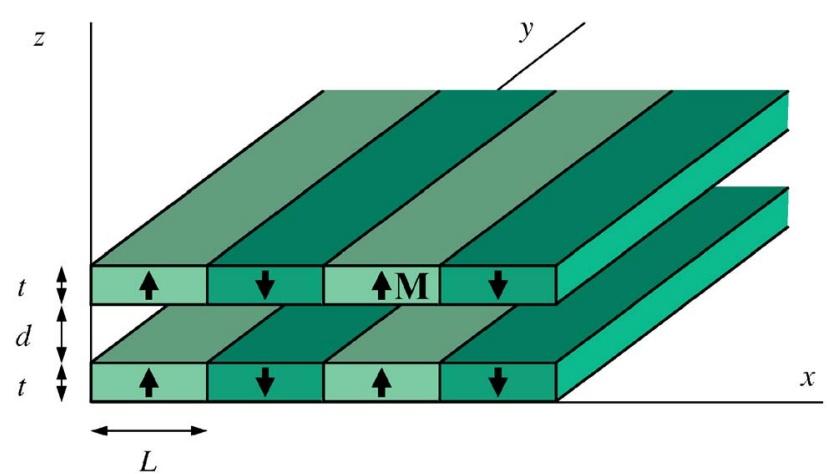

FIG. 1. (Color online) A bilayer structure representing two ferromagnetic films with a stripe domain structure separated by a nonmagnetic spacer layer.

$$
L^{2}=\frac{\sigma t}{1.7 M^{2}}
$$

The proportionality between the square of the domain width and the thickness of a film was indeed observed experimentally (see, e.g., Ref. 34).

When the domain width becomes much larger than the film thickness, Kittel's law does not hold (see, e.g., Ref. 35) and a more accurate account for the magnetostatic energy is necessary. In magnetic multilayers there is an additional contribution to the magnetostatic energy of a magnetic film coming from the stray fields produced by other magnetic films. ${ }^{36}$ This contribution has to be taken into account to predict the effect of the film thickness and the separation between the magnetic films on the domain size in a magnetic multilayer.

Here, we consider a bilayer structure consisting of two ferromagnetic layers of thickness $t$ separated by a nonmagnetic spacer layer of thickness $d$ (see Fig. 1). We assume that perpendicular anisotropy favors the magnetization perpendicular to the plane within each ferromagnetic layer. We consider the domain structure in the form of magnetic stripes of width $L$ with alternating magnetization in the $x$ direction, as shown in Fig. 1. The magnetic domains in the two films are aligned parallel due to the magnetostatic coupling and the IEC, both of which favor this alignment. We assume that the magnetization changes abruptly by $180^{\circ}$ from one stripe domain to the next, i.e., we neglect the variation of magnetization within the domain wall due to the domain width being much larger than the domain wall width. This may seem to be an oversimplification, but this approximation for the domain wall profile was shown in Ref. 33 to produce excellent agreement with experimental data.

The total magnetostatic energy density of the system per film can be expressed in the form

$$
E_{M}=E_{M}^{(0)}+E_{M}^{(1)},
$$

where

$$
E_{M}^{(0)}=\frac{16 M^{2} L}{\pi^{2} t} \sum_{n=1,3,5 \ldots}^{\infty} \frac{1}{n^{3}}\left(1-e^{-k_{n}^{x} t}\right)
$$

is the magnetostatic intralayer energy per unit volume per film due to the self-interaction of each magnetic layer, 


$$
E_{M}^{(1)}=-\frac{8 M^{2} L}{\pi^{2} t} \sum_{n=1,3,5 \ldots}^{\infty} \frac{1}{n^{3}} e^{-k_{n}^{x} d}\left(1-e^{-k_{n}^{x} t}\right)^{2}
$$

is the magnetostatic interlayer energy density due to the interaction between the layers, and $k_{n}^{x}=\pi n / L$. These results are consistent with the previous calculations ${ }^{36}$ and are derived in the Appendix [see Eqs. (A25) and (A27)]. This domain configuration constitutes a limiting case of a more complex domain structure, described in Sec. IV, which is formulated by expanding the periodic magnetization in Fourier series along both the $x$ and $y$ directions.

In the limit of large separation between the magnetic films $(d \rightarrow \infty)$, the magnetostatic energy (2) is reduced to the intralayer energy $E_{M}^{(0)}$ of a single film of thickness $t$. When $L \rightarrow \infty$, the latter gives the magnetostatic energy of a uniformly perpendicular-magnetized film $E_{M}^{(0)}=2 \pi M^{2}$. When $L$ $\ll t$ we can neglect the exponential in the intralayer energy, which yields an expression consistent with Kittel's result. ${ }^{31}$ In the opposite limit of $d \rightarrow 0$ the magnetostatic energy (2) is reduced to the intralayer energy $E_{M}^{(0)}$ of a single film of thickness $2 t$.

As follows from Eq. (3), the magnetostatic intralayer energy decreases with decreasing domain width $L$, hence favoring a smaller domain size. The magnetostatic energy is competing with the domain wall energy which increases with decreasing domain width $L$ due to the increasing number of domain walls per unit area. We can write the domain wall energy of the system per unit volume per film as follows:

$$
E_{\mathrm{dw}}=\frac{\sigma}{L}
$$

where $\sigma$ the domain wall energy per unit area for a single domain wall. The latter is given by $\sigma=4 \sqrt{A K}$, where $A$ and $K$ are the exchange stiffness constant and the uniaxial perpendicular anisotropy constant, respectively.

The equilibrium domain size is determined by the competition between the magnetostatic energy (2) and the domain wall energy (5) and can be found by minimizing the total energy $E=E_{M}+E_{d w}$ with respect to $L$. The dependence of the equilibrium domain size as a function of the thickness of the layers is shown in Fig. 2. Here and throughout this paper, we assume material specific parameters typical for CoPt multilayers for both magnetic films: $M=700$ $\mathrm{emu} / \mathrm{cm}^{3}, K=2 \times 10^{6} \mathrm{erg} / \mathrm{cm}^{3}$, and $A=1 \times 10^{-6} \mathrm{erg} / \mathrm{cm}$. We see that for small thickness $t$ the equilibrium domain size rapidly increases implying that the system has a tendency to turn into a single domain state. This is reflected in the energy $E$ plotted in the inset of Fig. 2 as a function of $L$ demonstrating that the energy minima become very shallow when the films are very thin. On the other hand, as thickness increases, the domain size decreases and the energy minima become well defined. This behavior is a strong departure from Kittel's law which predicts that the domain width is scaled as a square root of film thickness. ${ }^{31}$ Kittel's scaling is recovered at film thickness of the order of $100 \mathrm{~nm}$ and larger for which the domain width $L$ becomes comparable or smaller than $t$.

In the case of a single ferromagnetic film, the magneto-

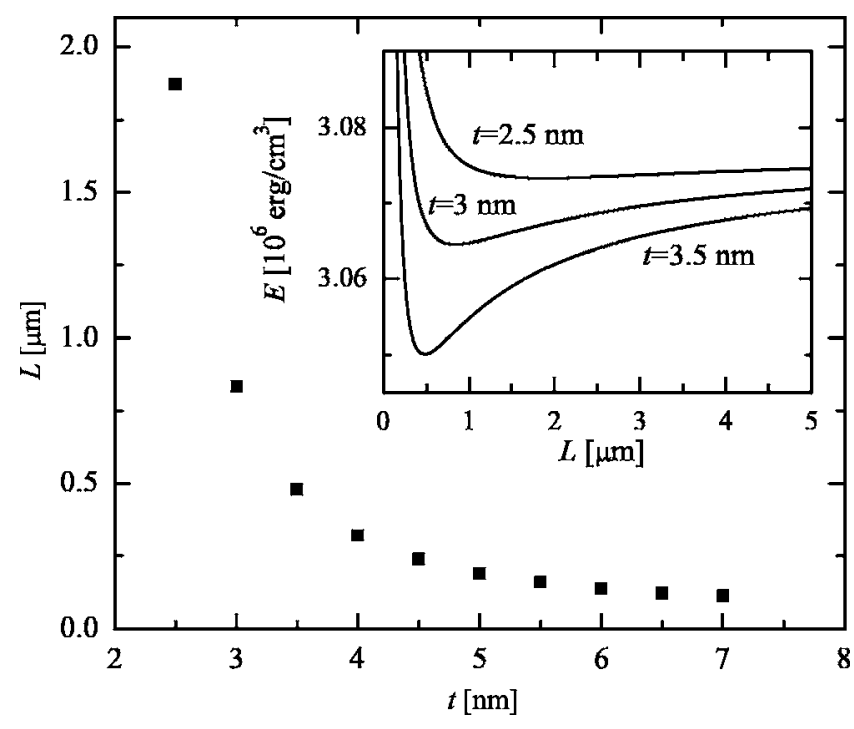

FIG. 2. The dependence of equilibrium domain size on the layer thickness $t$ for the interlayer separation $d=1 \mathrm{~nm}$. The inset shows the energy of the system as a function of the domain width $L$ for several values of $t$.

static energy determining the domain width is given solely by the magnetostatic intralayer energy $E_{M}^{(0)}$. The additional magnetostatic interlayer interaction energy $E_{M}^{(1)}$ decreases the overall magnetostatic energy and, in fact, makes smaller equilibrium domain size more favorable. This can be seen in Fig. 3 where the equilibrium domain size is plotted as a function of the interlayer separation $d$. In addition, it leads to deeper minima, corresponding to more stable domains. This effect is significant even for large separations between layers, as seen in Fig. 3, so that the domain width increases quite dramatically with increasing $d$ [and therefore decreasing $E_{M}^{(1)}$ in absolute magnitude]. Only when the separation between the layers becomes of the order of the domain size of the single layers does the magnetostatic interlayer interaction become negligible. This, however, occurs in our case for unre-

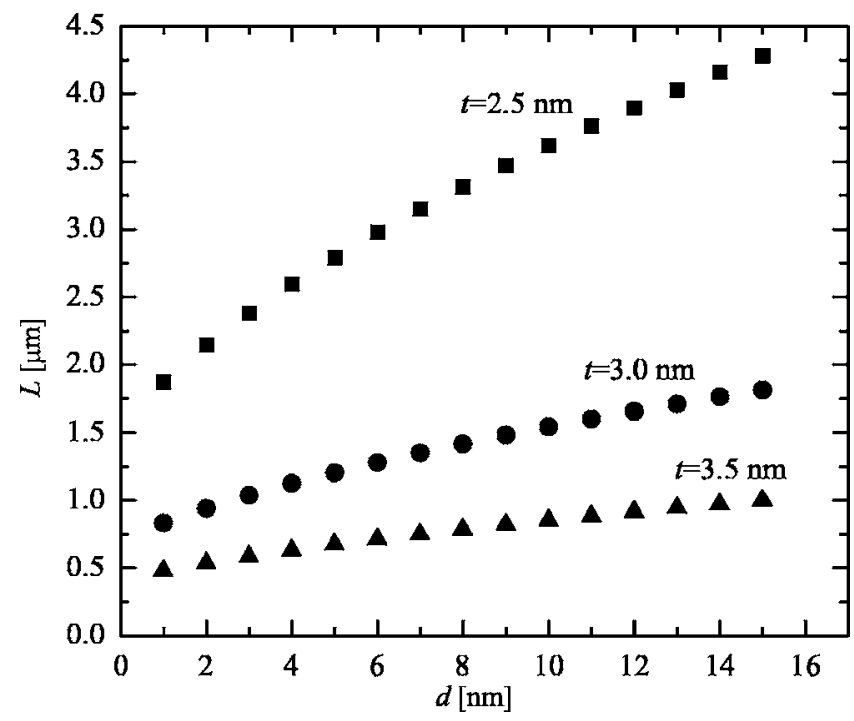

FIG. 3. The dependence of equilibrium domain size as a function of the distance between layers $d$ calculated for three different thicknesses: $t$ $=2.5 \mathrm{~nm}$ (squares), $t=3.0 \mathrm{~nm}$ (circles), and $t=3.5 \mathrm{~nm}$ (triangles). 


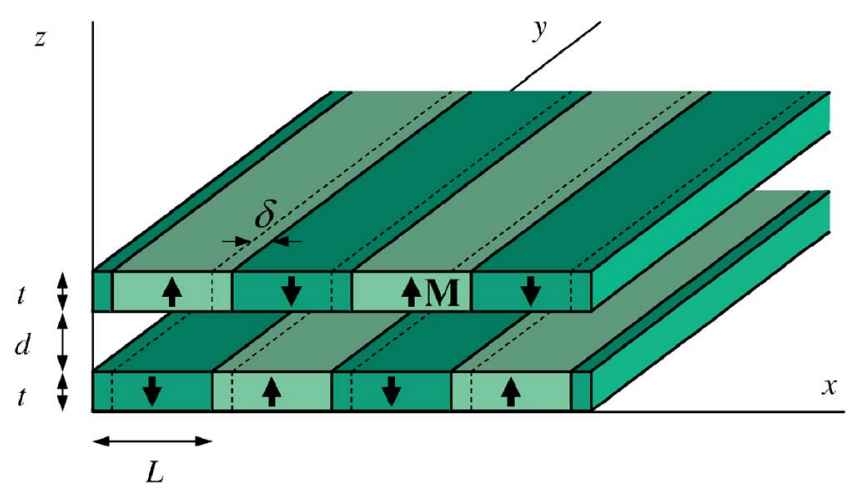

FIG. 4. (Color online) Antiferromagnetically coupled bilayer with a finite domain overlap. The dashed lines indicate the domain overlap regions of thickness $\delta$.

alistically large spacer thickness. Therefore, we find that the magnetostatic interaction energy has an important influence on the domain size.

\section{DOMAIN OVERLAP IN ANTIFERROMAGNETICALLY COUPLED BILAYERS}

In the presence of antiferromagnetic exchange coupling between magnetic films with perpendicular anisotropy, the domain structure exhibits an interesting behavior. It was found that $\mathrm{CoPt}$ films exchange coupled antiferromagnetically through $\mathrm{Ru}$ (Ref. 32) and $\mathrm{NiO}$ (Refs. 20 and 33) spacer layers exhibit a relative shift between the domains of the two magnetic layers. This behavior was explained by the competition between the antiferromagnetic exchange interaction and the magnetostatic coupling making the overlap energetically favorable. ${ }^{32,33}$ Here, we generalize the model introduced in the previous section to obtain additional insight into this phenomenon. In particular, we will show that there is a critical film thickness below which the domain overlap becomes energetically unfavorable.

We assume that there is an antiferromagnetic exchange coupling uniform throughout the surface of the bilayer and is characterized by the energy per unit area $J$. This interaction is much stronger than the magnetostatic interlayer coupling resulting largely in the antiparallel alignment of magnetic domains with some overlap $\delta$ produced by the magnetostatic coupling. This leads to the stripe domain structure shown in Fig. 4.

The domain overlap does not affect the magnetostatic intralayer energy or the domain wall energy, given by Eqs. (3) and (5). However, it has an important impact on both the magnetostatic interlayer interaction energy and the interlayer exchange energy. The magnetostatic energy per unit volume per film due to the interaction between the layers, derived as a limiting case of the complex domain configuration described in Sec. IV, is presented in the Appendix [see Eq. (A26)] and takes the form

$$
E_{M}^{(1)}=\frac{8 M^{2} L}{\pi^{2} t} \sum_{n=1,3,5 \ldots}^{\infty} \frac{1}{n^{3}} \cos \left(k_{n}^{x} \delta\right) e^{-k_{n}^{x} d}\left(1-e^{\left.-k_{n}^{x}\right)^{2}} .\right.
$$

This energy is reduced to Eq. (4) for $\delta=L$ which corresponds to perfect parallel alignment of the domains in the upper and

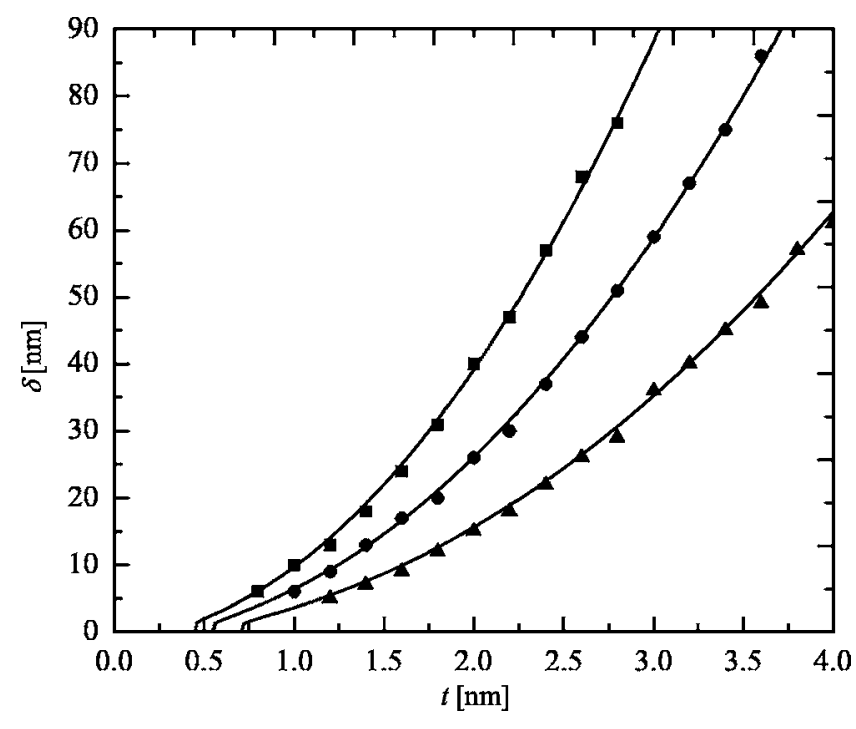

FIG. 5. The overlap as a function of the magnetic layer thickness $t$ for $J$ $=0.02 \mathrm{erg} / \mathrm{cm}^{2}$ (squares), $J=0.03 \mathrm{erg} / \mathrm{cm}^{2}$ (circles), and $J=0.05 \mathrm{erg} / \mathrm{cm}^{2}$ (triangles). The symbols show results of numerical calculations and solid lines are obtained from Eq. (10) showing excellent agreement. Parameters used: $L=1 \mu \mathrm{m}, d=1 \mathrm{~nm}$.

lower films. The interlayer exchange energy per unit volume per film is

$$
E_{\mathrm{IEC}}=\frac{J}{2 t}\left(\frac{2 \delta}{L}-1\right)
$$

where the interlayer exchange coupling constant $J$ is assumed to be positive for antiferromagnetic coupling. $E_{\mathrm{IEC}}$ is minimum when $\delta=0$, i.e., for perfect antiparallel alignment of magnetization. It is the competition between the energies (6) and (7) that yields a nonzero equilibrium value for $\delta$.

Figure 5 shows the calculated domain overlap $\delta$, as a function of layer thickness $t$ for three values of the interlayer exchange coupling $J=0.02,0.03$, and $0.05 \mathrm{erg} / \mathrm{cm}^{2}$, typical for the coupling through $\mathrm{NiO}$ in $\mathrm{CoPt} / \mathrm{NiO} / \mathrm{CoPt}$ systems. ${ }^{17,18}$ This calculation was performed by minimizing numerically the energy $E=E_{M}+E_{\mathrm{IEC}}$. In the calculation we fixed the value of the domain width $(L=1 \mu \mathrm{m})$ because we found that $\delta$ is insensitive to $L$ for typical domain sizes, making the domain wall energy $E_{\mathrm{dw}}$ a constant value. Two main features are evident from Fig. 5. First, there is a critical thickness below which the domain overlap disappears. Second, above the critical thickness the overlap increases with thickness of ferromagnetic films $t$ and decreases with the antiferromagnetic exchange coupling constant $J$.

This behavior can be understood from the asymptotic behavior of the energy $E=E_{M}+E_{\mathrm{IEC}}$ when $L \rightarrow \infty$. As follows from Eqs. (6) and (7), in this limit the derivative of the energy $E$ with respect to $\delta$ can be written as

$$
\frac{\partial E}{\partial \delta}=-\frac{8 M^{2} \pi t}{L^{2}} \sum_{n=1,3,5 \ldots}^{\infty} \sin \left(k_{n}^{x} \delta\right) e^{-k_{n}^{x} d}+\frac{J}{t L} .
$$

Replacing the summation by integration and taking the integral we find that 


$$
\frac{\partial E}{\partial \delta}=-\frac{4 M^{2} t}{L} \frac{\delta}{\delta^{2}+d^{2}}+\frac{J}{t L}
$$

Equating this energy to zero leads to the equilibrium domain overlap given by

$$
\delta=\frac{2 M^{2} t^{2}}{J}+\sqrt{\left(\frac{2 M^{2} t^{2}}{J}\right)^{2}-d^{2}} .
$$

It is evident from this equation that when $2 M^{2} t \gg J d$ the domain overlap $\delta$ follows a simple relationship:

$$
\delta=\frac{4 M^{2} t^{2}}{J} \text {. }
$$

This result is consistent with that derived previously in Ref. 33 within a model of a single domain wall which was used to explain experimental data for $\mathrm{CoPt} / \mathrm{NiO} / \mathrm{CoPt}$ multilayers. ${ }^{37}$ The fact that for not too small layer thickness the domain overlap is proportional to $t^{2}$ and inversely proportional to the antiferromagnetic exchange constant $J$ is evident from Fig. 5 . Here, our numerical calculations (symbols) and the results obtained from Eq. (10) (solid curves) demonstrate excellent agreement.

Equation (10) predicts that there is a critical thickness,

$$
t_{c}=\sqrt{\frac{J d}{2 M^{2}}},
$$

below which the domain overlap disappears because no real solution of Eq. (9) exists. The presence of a critical thickness is evident from Fig. 5. For $d=1 \mathrm{~nm}$ and $J=0.02 \mathrm{erg} / \mathrm{cm}^{2}$, we find that the critical thickness is relatively small, $t_{c}$ $=0.45 \mathrm{~nm}$. This value is, however, expected to be enhanced for a larger magnitude of the coupling $J$ according to Eq. (12). For example, for $d=1 \mathrm{~nm}$ and $J=2 \mathrm{ergs} / \mathrm{cm}^{2}$, which is not unrealistic for magnetic multilayers (see, e.g., Ref. 4), the critical thickness would be $t_{c}=4.5 \mathrm{~nm}$.

The physical origin of the critical thickness follows from the mechanism producing the domain overlap. This overlap arises due to the competition between the magnetostatic interaction and the interlayer exchange coupling: the magnetostatic interaction favors parallel alignment whereas the exchange interaction prefers antiparallel alignment of the domains. On average, the interlayer exchange coupling dominates the magnetostatic interaction and if the two were homogeneous over the surface the domains would align perfectly antiparallel with no overlap. However, the magnetostatic coupling is strongly inhomogeneous over the surface due to the stray fields localized in the vicinity of the domain walls, ${ }^{33}$ that makes it energetically favorable to produce a small shift $\delta$ between the antiparallel aligned domains to reduce the magnetostatic energy. However, when the thickness of magnetic films decreases the degree of inhomogeneity in the distribution of the magnetostatic energy density becomes smaller. At a certain critical thickness, the localization of this energy near domain walls becomes insufficient to stabilize the relative domain shift.

A similar effect occurs with increasing separation between magnetic layers. For a given film thickness $t$, there is a critical distance between magnetic films,

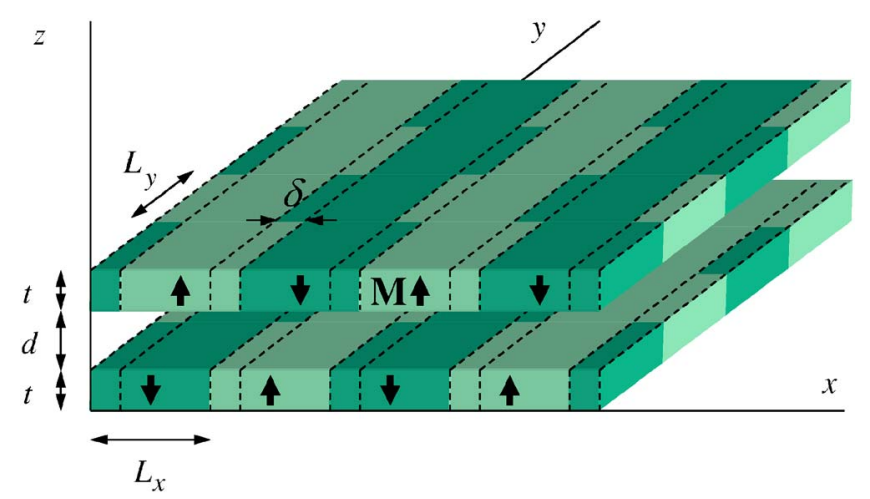

FIG. 6. (Color online) Antiferromagnetically coupled bilayer with a finite domain overlap. Dashed lines indicate the domain overlap regions with a "tiger tail" pattern produced along the overlap stripes.

$$
d_{c}=\frac{2 M^{2} t^{2}}{J}
$$

above which perfectly antiparallel alignment of magnetic domains becomes energetically more favorable. For parameters $J=0.02 \mathrm{erg} / \mathrm{cm}^{2}$ and $t=1 \mathrm{~nm}$, we find the critical distance to be approximately $5 \mathrm{~nm}$. According to Eq. (13) it decreases inversely proportional to $J$, and hence may be much smaller for a large antiferromagnetic IEC.

\section{IV. "TIGER TAIL" PATTERN IN THE OVERLAP REGION}

It was found experimentally that in addition to the overlap there is an additional superstructure in the overlap region, namely, the magnetization reverses periodically, producing a tiger tail pattern. ${ }^{32,33}$ To examine this, we generalize our model of stripe domains by introducing a periodic domain pattern in the overlap region, as shown in Fig. 6. Here, $2 L_{y}$ is the period in the direction along the overlap region.

The magnetostatic energy is found by expanding the magnetization in Fourier series in both $x$ and $y$ directions, as indicated previously. The detailed derivation is presented in the Appendix. The magnetostatic intralayer and interlayer energies are given by Eqs. (A21) and (A24), respectively. The reduction in magnetostatic energy gained by the development of the tiger tail pattern must compete against the energy due to the formation of additional domain walls in the overlap region, making the domain wall energy per unit volume per film

$$
E_{\mathrm{dw}}=\frac{\sigma}{L_{x}}+\frac{\sigma \delta}{L_{x} L_{y}} .
$$

We note that, rigorously in order to find the equilibrium value of $L_{y}$, we need to minimize the total energy with respect to $\delta$ and $L_{y}$ simultaneously. For the purposes of illustration, however, we consider the total energy as a function of $L_{y}$ assuming that all the other parameters are fixed. Therefore, the interlayer exchange coupling energy, given by Eq. (7), does not play a role when minimizing the total energy with respect to $L_{y}$, and apart from determining the fixed value of $\delta$ we choose using Eq. (10). 


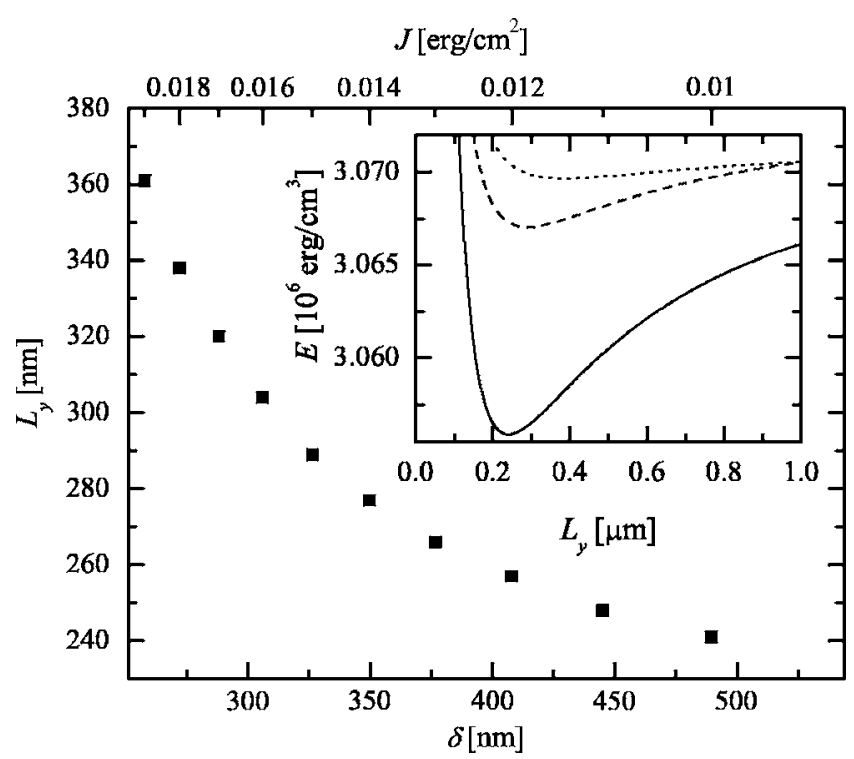

FIG. 7. The equilibrium domain period along the overlap region, $L_{y}$, as a function of the domain overlap $\delta$. The corresponding interlayer exchange coupling $J$ determined by Eq. (10) is shown on the top axis. Inset: The energy of the system as a function of $L_{y}$ for several values $\delta$ corresponding to several values of $J$ (solid line, $J=0.01 \mathrm{erg} / \mathrm{cm}^{2}$; dashed line, $J$ $=0.015 \mathrm{erg} / \mathrm{cm}^{2}$; dotted line, $\left.J=0.02 \mathrm{erg} / \mathrm{cm}^{2}\right)$.

We calculate numerically the total energy and find that it is energetically favorable for the system to produce a periodic domain structure in the overlap region because it further reduces the magnetostatic energy. The inset of Fig. 7 shows the total energy of the system as a function of $L_{y}$ for $t$ $=5 \mathrm{~nm}, d=1 \mathrm{~nm}$, and $L_{x}=1 \mu \mathrm{m}$ for three values of the domain overlap $\delta: 490,327$, and $245 \mathrm{~nm}$. Using Eq. (10), these correspond to interlayer exchange coupling values of $J$ $=0.01,0.015$, and $0.02 \mathrm{erg} / \mathrm{cm}^{2}$, respectively. As seen from the inset Fig. 7, there are well defined minima in the total energy indicating that the tiger tail structure reduces the energy.

It is also seen in Fig. 7 that the equilibrium $L_{y}$ decreases with increasing overlap $\delta$. In order to understand this trend, we can imagine the ferromagnetic overlap region as a onedimensional magnetic wire. Increasing $\delta$ corresponds to an increase in the size of the wire, thereby increasing the magnetostatic self-interaction of this wire. This increase in the magnetostatic energy can be minimized by the formation of smaller domains along the overlap region, corresponding to smaller $L_{y}$, at the expense of additional domain wall energy. This behavior is the one-dimensional analog of the dependence of domain size on thickness $t$ of the layer in the stripe domain model (see Fig. 2). We have also investigated the dependence of $L_{y}$ as a function of layer thickness and we found similar behavior to the dependence shown in Fig. 7.

Even though the tiger tail patterns have been observed experimentally, ${ }^{32,33}$ no experimental investigations were performed regarding the influence of the interlayer exchange coupling on the shape of these patterns produced along the overlap region. We hope that our theoretical predictions, in particular, the results shown in Fig. 7, will stimulate further experimental studies of the tiger tail features of the domain overlap phenomenon in antiferromagnetically coupled multilayers with perpendicular anisotropy.

\section{v. CONCLUSIONS}

We have investigated the influence of magnetostatic coupling on domain configurations in magnetic bilayer structures with perpendicular anisotropy. Assuming periodic stripe domain patterns with overlap regions resulting from the antiferromagnetic exchange coupling and additional periodic superstructure (tiger tail structure) along the overlap regions, as was observed in recent experiments, we found an analytic expression for the magnetostatic energy of the system. This allowed us to make quantitative conclusions about the domain size, the magnitude of the domain overlap, and the period of the tiger tail structure. We found that there is strong influence of the magnetostatic interlayer coupling on the equilibrium domain size. Increasing the separation between magnetic layers leads to the increase of the domain size due to the reduced magnetostatic coupling. For a bilayer exhibiting an antiferromagnetic exchange coupling consistent with previous results, we find the domain overlap due to the reduction of the magnetostatic energy in the vicinity of domain walls. We derived an analytic expression which shows how the width of the overlap depends on the magnitude of the antiferromagnetic interlayer exchange coupling, the magnetic layer thickness, and the separation between the magnetic layers. We predicted that there is a critical layer thickness and a critical distance between the layers beyond which the overlap disappears. Finally, we find that it is energetically favorable for the bilayer system to produce a periodic tiger tail structure along the overlap region to further reduce the magnetostatic energy. We hope that our results stimulate further experimental studies of magnetically coupled multilayers to verify our predictions.

\section{ACKNOWLEDGMENTS}

This work was supported the NSF-MRSEC (Grant No. DMR-0213808). We thank Andrew Baruth and Shireen Adenwalla for helpful discussions.

\section{APPENDIX}

Consider a bilayer structure consisting of two ferromagnetic films of thickness $t$ with perpendicular anisotropy separated by distance $d$ (see Fig. 6). The domain structure of each film consists of periodic stripe domains of width $L_{x}$. The layers are displaced from perfect antiferromagnetic alignment by $\delta$. Within the overlap regions there is an additional superstructure with periodically alternating magnetization of period $2 L_{y}$ (a tiger tail structure), as shown in Fig. 6. We calculate the magnetostatic energy of the system following the approach used in Ref. 38. The scalar magnetic potential produced by the bottom ferromagnetic film is given by

$$
\begin{aligned}
\Phi_{\mathrm{bot}}(\mathbf{r}) & =\int_{0}^{t} d z^{\prime} \int_{-\infty}^{\infty} d x^{\prime} \int_{-\infty}^{\infty} d y^{\prime} \frac{M\left(x^{\prime}, y^{\prime}\right)\left(z-z^{\prime}\right)}{\left|\mathbf{r}-\mathbf{r}^{\prime}\right|^{3}} \\
& =\int_{0}^{t} I\left(x, y, z-z^{\prime}\right) d z^{\prime},
\end{aligned}
$$

where $\mathbf{r}=(x, y, z), \mathbf{r}^{\prime}=\left(x^{\prime}, y^{\prime}, z^{\prime}\right)$ and, 


$$
\begin{aligned}
& I\left(x, y, z-z^{\prime}\right) \\
& =\int_{-\infty}^{\infty} d x^{\prime} \int_{-\infty}^{\infty} d y^{\prime} M\left(x^{\prime}, y^{\prime}\right) \Psi\left(x-x^{\prime}, y-y^{\prime}, z-z^{\prime}\right), \\
& \Psi(\mathbf{r})=\frac{z}{r^{3}} .
\end{aligned}
$$

It is convenient to rewrite the potential in terms of Fourier components of the magnetization. Using the convolution theorem we obtain

$$
\widetilde{I}\left(k_{x}, k_{y}\right)=2 \pi \widetilde{\Psi}\left(k_{x}, k_{y}\right) \widetilde{M}\left(k_{x}, k_{y}\right),
$$

where $\widetilde{\Psi}$ and $\tilde{M}$ denotes the Fourier transform of $\Psi$ and $M$, respectively. It can be shown that

$$
\begin{aligned}
\widetilde{\Psi}\left(k_{x}, k_{y}\right) & \equiv \frac{1}{2 \pi} \int_{-\infty}^{\infty} d x \int_{-\infty}^{\infty} d y e^{i k_{x} x} e^{i k_{y} y} \Psi(x, y) \\
& =e^{-\sqrt{k_{x}^{2}+k_{y}^{2}}\left|z-z^{\prime}\right|} \operatorname{sgn}\left(z-z^{\prime}\right) .
\end{aligned}
$$

In order to calculate the Fourier transform of the magnetization, we use the fact that it is a periodic function in the $x$ direction, so it can be expanded in the Fourier series

$$
M_{\mathrm{bot}}(x, y)=\sum_{n=-\infty}^{\infty} a_{n}(y) e^{i k_{n}^{x} x},
$$

where $k_{n}^{x} \equiv n \pi / L_{x}$ and

$$
a_{n}(y)=\frac{1}{2 L_{x}} \int_{0}^{2 L_{x}} M_{\mathrm{bot}}(x, y) e^{-i k_{n}^{x} x} d x .
$$

The magnetization of the bottom layer is given by

$$
M_{\mathrm{bot}}(x, y)= \begin{cases}M, & x \in\left(0, L_{x}-\delta\right) \\ M(y), & x \in\left(L_{x}-\delta, L_{x}\right) \\ -M, & x \in\left(L_{x}, 2 L_{x}-\delta\right) \\ -M(y), & x \in\left(2 L_{x}-\delta, 2 L_{x}\right) .\end{cases}
$$

Therefore, the Fourier coefficients are nonzero only for odd $n$ and are given by

$$
a_{n}(y)=\frac{i}{n \pi}\left[M(y)\left(e^{i k_{n}^{x} \delta}-1\right)-M\left(1+e^{i k_{n}^{x} \delta}\right)\right] .
$$

Since magnetization is also a periodic function in the $y$ direction, we can expand $M(y)$ in Fourier series as well

$$
M(y)=\sum_{m=-\infty}^{\infty} b_{m} e^{i k_{m}^{y} y},
$$

where $k_{m}^{y} \equiv m \pi / L_{y}$ and $b_{m}$ are nonzero only for odd $m$ and are given by

$$
b_{m}=\frac{1}{2 L_{y}} \int_{0}^{2 L_{y}} M(y) e^{-i k_{m}^{y} y} d y=\frac{i M}{m \pi}\left(e^{-i m \pi}-1\right) .
$$

Thus,

$$
M(y)=\sum_{\substack{m=-\infty \\ m=\text { odd }}}^{\infty} \frac{i M}{m \pi}\left(e^{-i m \pi}-1\right) e^{i k_{m}^{y} y},
$$

and consequently according to Eq. (A9) we find

$$
a_{n}(y)=\frac{-i M}{n \pi}\left[\left(1+e^{i k_{n}^{x} \delta}\right)+2\left(e^{i k_{n}^{x} \delta}-1\right) \sum_{\substack{m=-\infty \\ m=o d d}}^{\infty} \frac{i}{m \pi} e^{i k_{m}^{y} y}\right] .
$$

Therefore, the magnetization of the bottom layer is

$$
\begin{aligned}
M_{\mathrm{bot}}(x, y)= & -\frac{i M}{\pi} \sum_{\substack{n=-\infty \\
n=\text { odd }}}^{\infty} \frac{e^{i k_{n}^{x} x}}{n}\left(e^{i k_{n}^{x} \delta}+1\right) \\
& +\frac{2 M}{\pi^{2}} \sum_{\substack{m, n=-\infty \\
m, n=\text { odd }}}^{\infty} \frac{e^{i k_{n}^{x} \delta}-1}{m n} e^{i k_{n}^{x} x} e^{i k_{m}^{y} y}
\end{aligned}
$$

As follows from Eq. (A14), the inverse Fourier transform of magnetization is given by

$$
\begin{aligned}
\tilde{M}\left(k_{x}, k_{y}\right)= & -2 i M \sum_{\substack{n=-\infty \\
n=\text { odd }}}^{\infty} \frac{e^{-i k_{n}^{x} \delta}+1}{n} \delta\left(k_{x}-k_{n}^{x}\right) \delta\left(k_{y}\right) \\
& +\frac{4 M}{\pi} \sum_{\substack{m, n=-\infty \\
m, n=\text { odd }}}^{\infty} \frac{e^{-i k_{n}^{x} \delta}-1}{m n} \delta\left(k_{x}-k_{n}^{x}\right) \delta\left(k_{y}-k_{m}^{y}\right) .
\end{aligned}
$$

Using Eqs. (A4), (A5), and (A15) we can evaluate (A2) to obtain $I\left(x, y, z-z^{\prime}\right)$

$$
\begin{aligned}
& I\left(x, y, z-z^{\prime}\right)=-2 i M \operatorname{sgn}\left(z-z^{\prime}\right) \sum_{\substack{n=-\infty \\
n=\text { odd }}}^{\infty} \frac{e^{i k_{n}^{x} x}}{n}\left(e^{i k_{n}^{x} \delta}+1\right) \\
& \times e^{-\left|k_{n}^{x}\right|\left|z-z^{\prime}\right|}+\frac{4 M}{\pi} \operatorname{sgn}\left(z-z^{\prime}\right) \\
& \times \sum_{m, n=-\infty}^{\infty} \frac{e^{i k_{n}^{x} x} e^{i k_{m}^{y} y}}{m n}\left(e^{i k_{n}^{x} \delta}-1\right) \\
& m, n=\text { odd } \\
& \times e^{-\left|z-z^{\prime}\right| \sqrt{\left(k_{n}^{x}\right)^{2}+\left(k_{m}^{y}\right)^{2}}} .
\end{aligned}
$$

Now we can calculate the potential (A1) produced by the bottom layer. For $z>t$ we find 


$$
\Phi_{\text {bot }}^{(1)}=\frac{-2 i M L_{x}}{\pi} \sum_{\substack{n=-\infty \\ n=\text { odd }}}^{\infty} \frac{e^{i k_{n}^{x} x}}{n|n|}\left(e^{i k_{n}^{x} \delta}+1\right)\left[e^{-k_{n}^{x}(z-t)}-e^{-k_{n}^{x} z}\right]+\frac{2 M}{\pi} \sum_{\substack{m, n=-\infty \\ m, n=\text { odd }}}^{\infty} \frac{e^{i k_{n}^{x} x} e^{i k_{m}^{y} y}}{m n} \frac{\left(e^{i k_{n}^{x} \delta}-1\right)}{\sqrt{\left(k_{n}^{x}\right)^{2}+\left(k_{m}^{y}\right)^{2}}}\left[e^{-(z-t) \sqrt{\left(k_{n}^{x}\right)^{2}+\left(k_{m}^{y}\right)^{2}}}-e^{-z \sqrt{\left(k_{n}^{x}\right)^{2}+\left(k_{m}^{y}\right)^{2}}}\right] .
$$

For $0<z<t$ we have

$$
\Phi_{\mathrm{bot}}^{(0)}=\frac{-2 i M L_{x}}{\pi} \sum_{\substack{n=-\infty \\ \text { odd }}}^{\infty} \frac{e^{i k_{n}^{x} x}}{n|n|}\left(e^{i k_{n}^{x} \delta}+1\right)\left[e^{-k_{n}^{x}(t-z)}-e^{-k_{n}^{x}}\right]+\frac{4 M}{\pi} \sum_{\substack{m, n=-\infty \\ m, n=\text { odd }}}^{\infty} \frac{e^{i k_{n}^{x} x} e^{i k_{m}^{y} y}}{m n} \frac{\left(e^{i k_{n}^{x} \delta}-1\right)}{\sqrt{\left(k_{n}^{x}\right)^{2}+\left(k_{m}^{y}\right)^{2}}}\left[e^{-(t-z) \sqrt{\left(k_{n}^{x}\right)^{2}+\left(k_{m}^{y}\right)^{2}}}-e^{-z \sqrt{\left(k_{n}^{x}\right)^{2}+\left(k_{m}^{y}\right)^{2}}}\right] .
$$

Given the magnetostatic scalar potential we are now able to calculate the magnetostatic energy. We split the total magnetostatic energy into the energy associated with the interaction within the layers $E_{M}^{(0)}$ and the energy associated with the interaction between the layers $E_{M}^{(1)}$. The magnetostatic intralayer energy per unit volume per film is given by

$$
E_{M}^{(0)}=\frac{1}{2} \frac{1}{4 L_{x} L_{y} t} \int_{0}^{2 L_{x}} d x \int_{t+d}^{2 t+d} d z \int_{0}^{2 L_{y}} d y \Phi_{\mathrm{bot}}^{(0)}(x, y, z) \rho_{\mathrm{bot}}(x, y, z)
$$

Here, we take into account that the two films are identical and hence the intralyer energy per unit volume needs to be calculated within one film only. The factor $\frac{1}{2}$ is due to the double counting. $\rho_{\text {bot }}(x, y, z)$ is the magnetic charge density of the bottom layer which is

$$
\rho_{\mathrm{bot}}=\left\{\begin{array}{l}
-M \delta(z)+M \delta(z-t), \quad x \in\left(0, L_{x}-\delta\right) ; \\
+M \delta(z)-M \delta(z-t), \quad x \in\left(L_{x}, 2 L_{x}-\delta\right) ;\left\{\begin{array}{l}
x \in\left(L_{x}-\delta, L_{x}\right) \\
y \in\left(0, L_{y}\right) \\
x \in\left(L_{x}-\delta, L_{x}\right) \\
y \in\left(L_{y}, 2 L_{y}\right)
\end{array} ;\left\{\begin{array}{l}
x \in\left(2 L_{x}-\delta, 2 L_{x}\right) \\
y \in\left(L_{y}, 2 L_{y}\right)
\end{array} ; \begin{array}{l}
x \in\left(2 L_{x}-\delta, 2 L_{x}\right) \\
y \in\left(0, L_{y}\right)
\end{array} .\right.\right.
\end{array}\right.
$$

Evaluating the integral in (A19) we obtain

$$
E_{M}^{(0)}=\frac{16 M^{2} L_{x}}{t \pi^{2}} \sum_{n=1,3,5, \ldots} \frac{\cos ^{2}\left(k_{n}^{x} \delta / 2\right)}{n^{3}}\left(1-e^{-k_{n}^{x} t}\right)+\frac{128 M^{2}}{t \pi^{3}} \sum_{\substack{n=1,3,5, \ldots \\ m=1,3,5, \ldots}} \frac{\sin ^{2}\left(k_{n}^{x} \delta / 2\right)}{m^{2} n^{2}} \frac{\left(1-e^{-t \sqrt{\left(k_{n}^{x}\right)^{2}+\left(k_{m}^{y}\right)^{2}}}\right)}{\sqrt{\left(k_{n}^{x}\right)^{2}+\left(k_{m}^{y}\right)^{2}}}
$$

The magnetostatic interlayer energy per unit volume per film is given by

$$
E_{M}^{(1)}=\frac{1}{2 t} \frac{1}{4 L_{x} L_{y}} \int_{0}^{2 L_{x}} d x \int_{t+d}^{2 t+d} d z \int_{0}^{2 L_{y}} d y \Phi_{\mathrm{bot}}^{(1)}(x, y, z) \rho_{\mathrm{top}}(x, y, z)
$$

Here, $\rho_{\text {top }}(x, y, z)$ is the magnetic charge density of the top layer which is

$$
\rho_{\text {top }}=\left\{\begin{array}{l}
+M \delta(z)-M \delta(z-t), \quad x \in\left(0, L_{x}-\delta\right) ; \\
-M \delta(z)+M \delta(z-t), \quad x \in\left(L_{x}, 2 L_{x}-\delta\right) ;\left\{\begin{array}{l}
x \in\left(L_{x}-\delta, L_{x}\right) \\
y \in\left(L_{y}, 2 L_{y}\right) \\
x \in\left(L_{x}-\delta, L_{x}\right) \\
y \in\left(0, L_{y}\right)
\end{array} ;\left\{\begin{array}{l}
x \in\left(2 L_{x}-\delta, 2 L_{x}\right) \\
y \in\left(0, L_{y}\right)
\end{array} ;\left\{\begin{array}{l}
x \in\left(2 L_{x}-\delta, 2 L_{x}\right) \\
y \in\left(L_{y}, 2 L_{y}\right)
\end{array} .\right.\right.\right.
\end{array}\right.
$$

Evaluating the integral in (A22) we find

$$
\begin{aligned}
E_{M}^{(1)}= & \frac{8 M^{2} L_{x}}{t \pi^{2}} \sum_{n=1,3,5, \ldots} \frac{\cos ^{2}\left(k_{n}^{x} \delta / 2\right)}{n^{3}}\left(e^{-k_{n}^{x} d}-2 e^{-k_{n}^{x}(t+d)}+e^{-k_{n}^{x}(2 t+d)}\right) \\
& -\frac{64 M^{2}}{t \pi^{3}} \sum_{\substack{n=1,3,5, \ldots \\
m=1,3,5}} \frac{\sin ^{2}\left(k_{n}^{x} \delta / 2\right)}{m^{2} n^{2} \sqrt{\left(k_{n}^{x}\right)^{2}+\left(k_{m}^{y}\right)^{2}}}\left(e^{-d \sqrt{\left(k_{n}^{x}\right)^{2}+\left(k_{m}^{y}\right)^{2}}}-2 e^{-(t+d) \sqrt{\left(k_{n}^{x}\right)^{2}+\left(k_{m}^{y}\right)^{2}}}+e^{-(2 t+d) \sqrt{\left(k_{n}^{x}\right)^{2}+\left(k_{m}^{y}\right)^{2}}}\right) .
\end{aligned}
$$


In the case when there is no superstructure along the $y$ direction we can obtain expressions for magnetostatic energies by letting $L_{y} \rightarrow \infty$. For the intralayer energy we obtain

$$
\lim _{L_{y} \rightarrow \infty} E_{M}^{(0)}=\frac{16 M^{2} L_{x}}{\pi^{2} t} \sum_{n=1,3,5, \ldots} \frac{1}{n^{3}}\left(1-e^{-k_{n}^{x} t}\right),
$$

which is obviously independent of $\delta$. For the interlayer energy we have

$$
\lim _{L_{y} \rightarrow \infty} E_{M}^{(1)}=\frac{8 M^{2} L_{x}}{\pi^{2} t} \sum_{n=1,3,5 \ldots} \frac{\cos \left(k_{n}^{x} \delta\right)}{n^{3}} e^{-k_{n}^{x} d}\left(1-e^{-k_{n}^{x} t}\right)^{2} .
$$

For perfect parallel alignment of the layers we just set $\delta$ $=L_{x}$ which leads to

$$
E_{M}^{(1)}=-\frac{8 M^{2} L_{x}}{\pi^{2} t} \sum_{n=1,3,5 \ldots} \frac{e^{-k_{n}^{x} d}}{n^{3}}\left(1-e^{-k_{n}^{x} t}\right)^{2} .
$$

${ }^{1}$ E. Y. Tsymbal and D. G. Pettifor, in Solid State Physics, edited by H. Ehrenreich and F. Spaepen (Academic, New York, 2001), Vol. 56, pp. 113-237.

${ }^{2}$ E. Y. Tsymbal, O. N. Mryasov, and P. R. LeClair, J. Phys.: Condens. Matter 15, R109 (2003).

${ }^{3}$ P. Bruno, Phys. Rev. B 49, 13231 (1994).

${ }^{4}$ M. D. Stiles, in Ultrathin Magnetic Structures III, edited by B. Heinrich and J. A. C. Bland (Springer, New York, 2005).

${ }^{5}$ P. Grünberg, R. Schreiber, Y. Pang, M. B. Brodsky, and H. Sowers, Phys. Rev. Lett. 57, 2442 (1986)

${ }^{6}$ S. S. P. Parkin, N. More, and K. P. Roche, Phys. Rev. Lett. 64, 2304 (1990).

${ }^{7}$ S. S. P. Parkin, Phys. Rev. Lett. 67, 3598 (1991).

${ }^{8}$ V. Grolier et al., Phys. Rev. Lett. 71, 3023 (1993).

${ }^{9}$ P. Bruno and C. Chappert, Phys. Rev. Lett. 67, 1602 (1991).

${ }^{10}$ D. M. Edwards, J. Mathon, R. B. Muniz, and M. S. Phan, Phys. Rev. Lett. 67, 493 (1991).

${ }^{11}$ C. Slonczewski, Phys. Rev. B 39, 6995 (1989).

${ }^{12}$ P. Bruno, Phys. Rev. B 52, 411 (1995).

${ }^{13}$ J. Faure-Vincent, C. Tiusan, C. Bellouard, E. Popova, M. Hehn, F. Montaigne, and A. Schuhl, Phys. Rev. Lett. 89, 107206 (2002).

${ }^{14}$ R. R. Gareev, L. L. Pohlmann, S. Stein, D. E. Bürgler, P. A. Grünberg, and M. Siegel, J. Appl. Phys. 93, 8038 (2003).

${ }^{15}$ M. Y. Zhuravlev, E. Y. Tsymbal, and A. V. Vedyayev, Phys. Rev. Lett. 94, 026806 (2005)
${ }^{16}$ T. Katayama, S. Yuasa, J. P. Velev, M. Y. Zhuravlev, S. S. Jaswal, and E. Y. Tsymbal, Appl. Phys. Lett. 89, 112503 (2006).

${ }^{17}$ Z. Y. Liu and S. Adenwalla, Phys. Rev. Lett. 91, 037207 (2003).

${ }^{18}$ Z. Y. Liu, L. Yue, D. J. Keavney, and S. Adenwalla, Phys. Rev. B 70, 224423 (2004)

${ }^{19}$ M. Y. Zhuravlev, E. Y. Tsymbal, and S. S. Jaswal, Phys. Rev. Lett. 92, 219703 (2004)

${ }^{20}$ A. Baruth, D. J. Keavney, J. D. Burton, K. Janicka, E. Y. Tsymbal, L. Yuan, S. H. Liou, and S. Adenwalla, Phys. Rev. B 74, 054419 (2006).

${ }^{21}$ L. Néel, C. R. Acad. Sci. 255, 1676 (1962).

${ }^{22}$ B. D. Schrag et al., Appl. Phys. Lett. 77, 2373 (2000).

${ }^{23}$ J. Moritz, F. Garcia, J. Toussaint, B. Dieny, and J.-P. Nozières, Europhys. Lett. 65, 123 (2004)

${ }^{24}$ S. Demokritov, E. Tsymbal, P. Grünberg, W. Zinn, and I. K. Schuller, Phys. Rev. B 49, R720 (1994); U. Rücker, S. Demokritov, E. Tsymbal, P. Grünberg, and W. Zinn, J. Appl. Phys. 78, 387 (1995); S. O. Demokritov, J. Phys. D 31, 925 (1998).

${ }^{25}$ E. Y. Tsymbal and D. G. Pettifor, J. Magn. Magn. Mater. 202, 163 (1999); E. Y. Tsymbal, K. D. Belashchenko, J. P. Velev, S. S. Jaswal, M. van Schilfgaarde, I. I. Oleynik, and D. A. Stewart, Prog. Mater. Sci. 52, 401 (2007).

${ }^{26}$ E. F. Fullerton, D. M. Kelly, J. Guimpel, I. K. Schuller, and Y. Bruynseraede, Phys. Rev. Lett. 68, 859 (1992); H. Kano, K. Kagawa, A. Suzuki, A. Okabe, K. Hayashi, and K. Asoa, Appl. Phys. Lett. 63, 2839 (1993); M. Suzuki and Y. Taga, Phys. Rev. B 52, 361 (1995); T. N. Todorov, E. Y. Tsymbal, and D. G. Pettifor, ibid. 54, R12685 (1996).

${ }^{27}$ L. Thomas, M. Samant, and S. Parkin, Phys. Rev. Lett. 84, 1816 (2000).

${ }^{28}$ W. Lew, S. Li, L. Lopez-Diaz, D. Hatton, and J. Bland, Phys. Rev. Lett. 90, 217201 (2003).

${ }^{29}$ R. Schäfer, R. Urban, D. Ullmann, H. Meyerheim, B. Heinrich, L. Schultz, and J. Kirschner, Phys. Rev. B 65, 144405 (2002).

${ }^{30}$ F. Garcia, F. Fettar, S. Auffret, B. Rodmacq, and B. Dieny, J. Appl. Phys. 93, 8397 (2003).

${ }^{31}$ C. Kittel, Phys. Rev. 70, 965 (1946).

${ }^{32}$ O. Hellwig, A. Berger, and E. E. Fullerton, Phys. Rev. Lett. 91, 197203 (2003).

${ }^{33}$ A. Baruth, L. Yuan, J. D. Burton, K. Janicka, E. Y. Tsymbal, S. H. Liou, and S. Adenwalla, Appl. Phys. Lett. 89, 202505 (2006).

${ }^{34}$ C. L. Dennis et al., J. Phys.: Condens. Matter 14, R1175 (2002).

${ }^{35}$ R. Gemperle, L. Murtinova, and V. Kambersky, Phys. Status Solidi A 158, 229 (1996).

${ }^{36}$ H. J. G. Draaisma and W. J. M. De Jonge, J. Appl. Phys. 62, 3318 (1987).

${ }^{37}$ Note that Eq. (11) differs by a factor of 2 from the similar result of Eq. (5) in Ref. 33. This can be accounted for by a different definition of the IEC coupling constant $J$, which is defined to be larger by a factor of 2 in Ref. 33 than in this work.

${ }^{38}$ E. Tsymbal, J. Magn. Magn. Mater. 130, L6 (1994); Appl. Phys. Lett. 77, $2740(2000)$ 